\title{
Supplemental Literature Review
}

This section is intended to provide the most current phase diagram data. Guidelines for the inclusion of new information in this section are (1) systems for which no phase diagrams are given in Binary Alloy Phase Diagrams,second edition; (2) complete diagrams that are substantially different from earlier versions published in Binary Alloy Phase Diagrams,second edition, the Bulletin of Alloy Phase Diagrams,or single-topic monographs; (3) partial diagrams that alter or clarify earlier versions in the above-mentioned publications; and (4) relevant new literature of interest.

Thermodynamic consistency of the new phase diagrams was checked based on phase rules, and the diagrams were modified if necessary. However, the diagrams and texts have not gone through the ordinary reviewing process, and the final evaluations may be carried out by relevant category editors of the Alloy Phase Diagram Program. For convenience, reaction tables and crystal structure data are added when new information is available.

H. Okamoto Supplemental Literature Review Editor 